\title{
Feasibility analysis of unique ID (UID) generation for personal authentication of valuable documents using dorsal hand vein pattern.
}

\author{
AMBADIYIL, S., JAYARAIAN, R., PILLAI, V.P.M., PRABHU, R.
}




\title{
Feasibility Analysis of Unique ID (UID) Generation for Personal Authentication of Valuable Documents Using Dorsal Hand Vein Pattern
}

\author{
Sajan Ambadiyil ${ }^{\mathrm{a}}$, Reema Jayarajan ${ }^{\mathrm{b}}$, V P Mahadevan Pillai ${ }^{\mathrm{c}}$ and Radhakrishna Prabhu ${ }^{\mathrm{d}}$ \\ ${ }^{a}$ Center for Development of Imaging Technology, Thiruvananthapuram-695027, Kerala, India \\ bJawaharlal College of Engineering and Technology, Palakkad-679301, Kerala, India \\ ${ }^{\circ}$ Department of Optoelectronics, University of Kerala, Thiruvananthapuram-695581, Kerala, India \\ ${ }^{\mathrm{d}}$ Robert Gordon University, School of Engineering, Aberdeen, United Kingdom
}

\begin{abstract}
Reliable personal authentication is a critical and vital obligation to the security in all the real-world applications. Nevertheless, biometric features are effectively used for the personal authentication, in some cases the criminal impersonation is an easy task. The reproducible attack in fingerprint system and cost prohibitive nature of iris and facial based system limit the vast implementation of the same for personal authentication. Hence, considering the cases of increasing identity theft, there is more reason than ever to ensure the reliable and cost-effective personal authentication. The vein biometric identification system has been gaining increased attention in recent years. Anatomically, the shape of the vein pattern at the dorsal hand is unique for each even for identical twins and remains stable for a period. The objective of this work is to conduct a feasibility analysis of unique ID generation using dorsal hand vein pattern as the biometric authentication system for preventing identity theft of the valuable documents. Images of the vein pattern from the dorsal side of the hand are tried to capture using a regular type Smartphone camera. Vein pattern thus captured is used to extract the unique features. Numerical values generated from such unique feature are encrypted and used to create a unique ID. This paper discusses about the method for the dorsal hand vein pattern capturing, its feature extraction, conversion to the unique ID and its feasibility to integrate to the valuable documents.
\end{abstract}

Keywords: Authentication, impersonation, dorsal hand vein, knuckle, maximum curvature method,

\section{INTRODUCTION}

In today's globally connected world, as the level of identity theft and security breaches are increasing in the fastest manner; the need for secure identification and authentication technologies is becoming apparent. The consequences of insecure authentication may lead to catastrophe, denial of service, loss of confidential data etc. Nevertheless, biometric features are effectively used for the personal authentication, in some cases the criminal impersonation is an easy task. The fingerprint is considered to be the most cost-effective method for personal identification, the credibility of such technology has been questioned due to the easily reproducible attacks [1]. Personal identification using iris is the unique and distinguishable human feature, the scanners to obtain the precise structure of the above traits are very costly. Facial recognition method does not need any expensive equipment but requires the costliest software for feature processing.

Now, the vascular biometrics offer several intrinsic advantages in comparison with other popular biometric modalities like fingerprint, iris, facial recognition etc. In vascular biometrics, the biological element used for identification is fully internal and not visible hence the security achieved is high. Blood veins are molded during the first eight weeks of gestation in a chaotic manner, influenced by the environment in a mother's womb. Even if the capillary structures grow and change, the vascular pattern is set at birth and do not change. Thus the geometry of these veins is found to be unique and universal [2] for all the persons each even for identical twins. In this study, we are only taking the dorsal hand vein pattern for the feasibility analysis of generating unique ID (UID) for preventing identity theft in the valuable documents. Fig. 1 shows the schematic representation of the dorsal hand vein pattern [3]. As shown in the figure, the vein pattern at the dorsal hand is inside the human body, so it is not possible to change or tamper the pattern and hard to be copied or captured by the intruders compared to the biometric traits like the fingerprint [4]. Moreover, the dorsal hand veins are not deeply placed, and hence these can be made visible with the help of a good image acquisition system and technique [5]. 


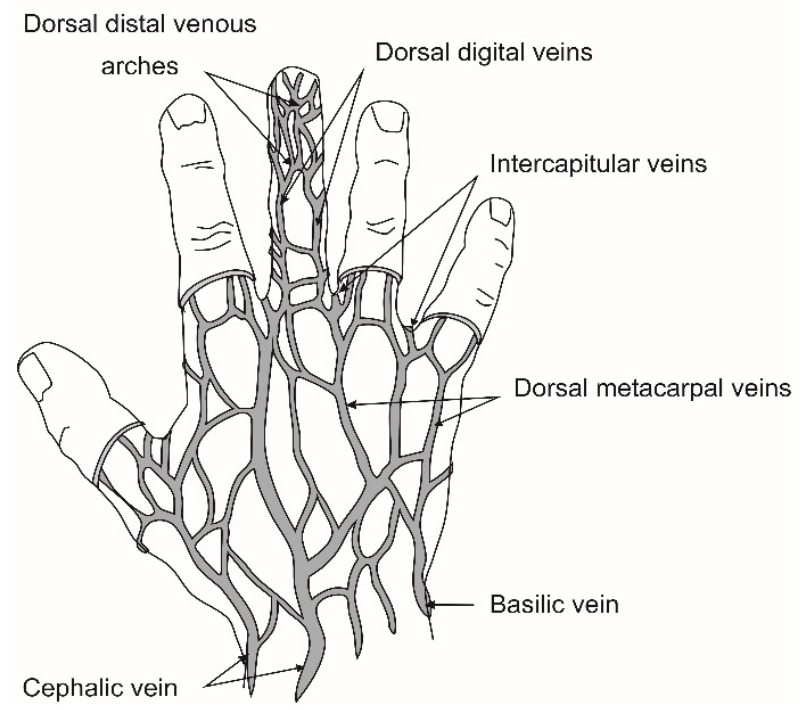

Fig.1 Schematic representation of the dorsal hand vein pattern

\section{THE PROPOSED METHOD}

In the proposed system, the unique pattern of the dorsal hand vein is exploited for generating unique ID (UID) for preventing identity theft of the valuable documents. From the unique pattern of the dorsal hand vein, the features are extracted, encrypted and converted into a QR code and is imprinted on the valuable document. This QR code can be verified for authentication of a valuable document possessed by the end user. A detailed block diagram for feature extraction and UID generation from dorsal hand vein pattern of an individual is shown in Fig. 2.

\subsection{Image acquisition}

Dorsal vein pattern is taken by keeping the hand in such a way that the skin on the back of the hand is taut, by clenching the fist. This will provide the skeleton of the hand as well as the vein pattern rigid and clear. The corresponding pattern is then picked up by a suitable image capturing system for further processing. In this study, the image of the dorsal hand portion is captured using a regular type mobile phone camera (13 MP). So far, high-cost IR imaging devices have been used on the majority of research conducted in vein pattern analysis for biometric applications as they produce high-quality images [6]. Recent work has focused on utilizing low-cost alternative imaging devices including standard webcams which have been modified to be sensitive to infrared wavelengths to enable imaging of vein patterns [7]. In this work, a mobile phone camera is used for image acquisition. We attempted to develop a cost-effective system including a simple image capturing device. Fig. 3(a) shows the dorsal hand vein pattern captured using a regular camera.

\subsection{Pre-processing}

The obtained image is then converted into the grayscale image and resized to convert into a common size. Image resizing increases the processing speed. Conversion into grey-scale reduces the image size from 24-bits in each pixel to 8-bits in each black and white pixel. The size of the resized image is fixed as 256 x 256 pixels. Bicubic interpolation is used while resizing the image. After resizing the image, a thresholding operation is done using Otsu thresholding method, which is a global thresholding method. This generates a binary image from the grey-scale image. In order to extract the feature point from the vain pattern, knuckle points of various fingers are to be identified first. In order to obtain the knuckle point from the acquired image canny edge detection algorithm is applied to the binarized image to reduce the amount of data in an image, by preserving the structural properties. In this algorithm, the image is blurred to remove noise by applying a Gaussian filter. Then, the edges in the images are marked by finding out, where the gradients in the image have larger magnitude. After this, the blurred edges in gradient images are converted into sharp edges by preserving all the local maxima in the gradient images and deleting the rest. Fig. 3(b) \& (c) show the binarized image and edge detected images. 


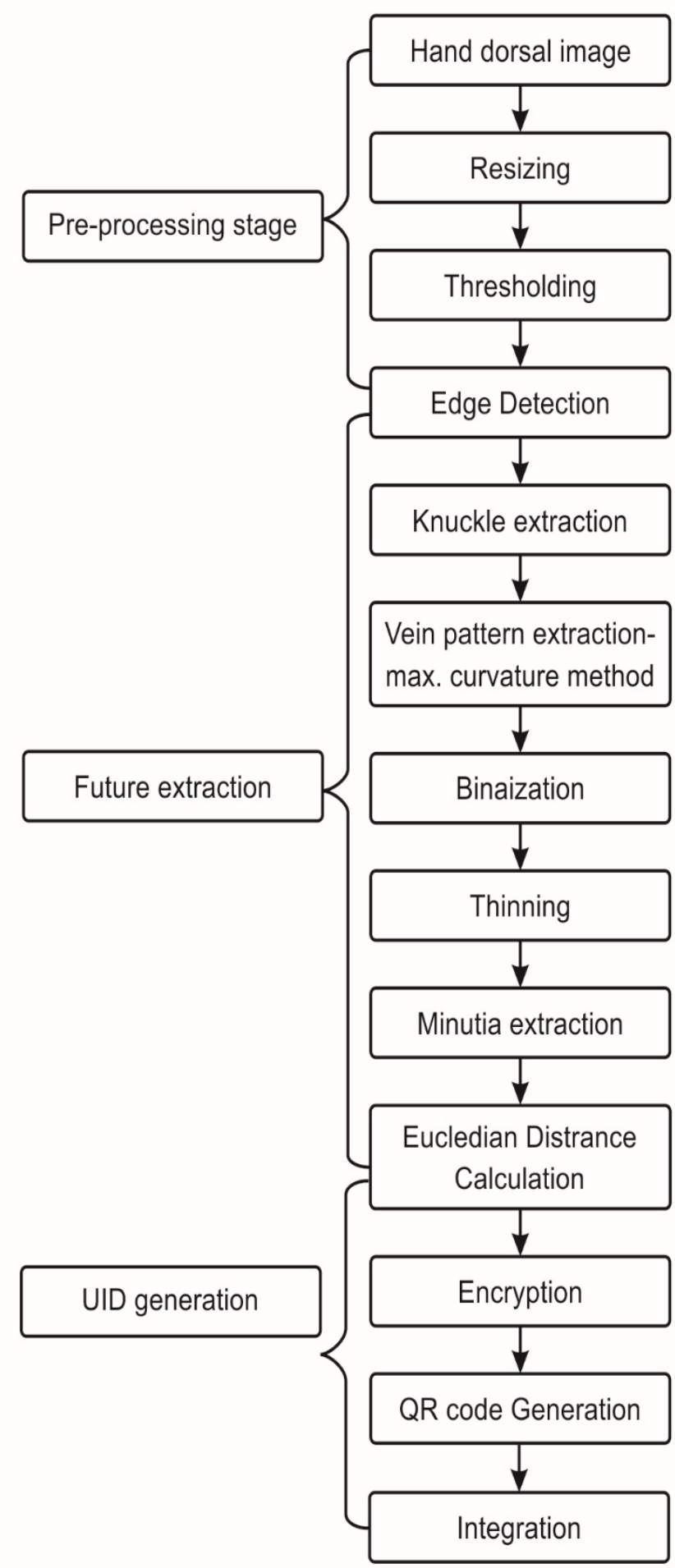

Fig. 2 The detailed block diagram for feature extraction and UID generation from dorsal hand vein pattern of an individual 

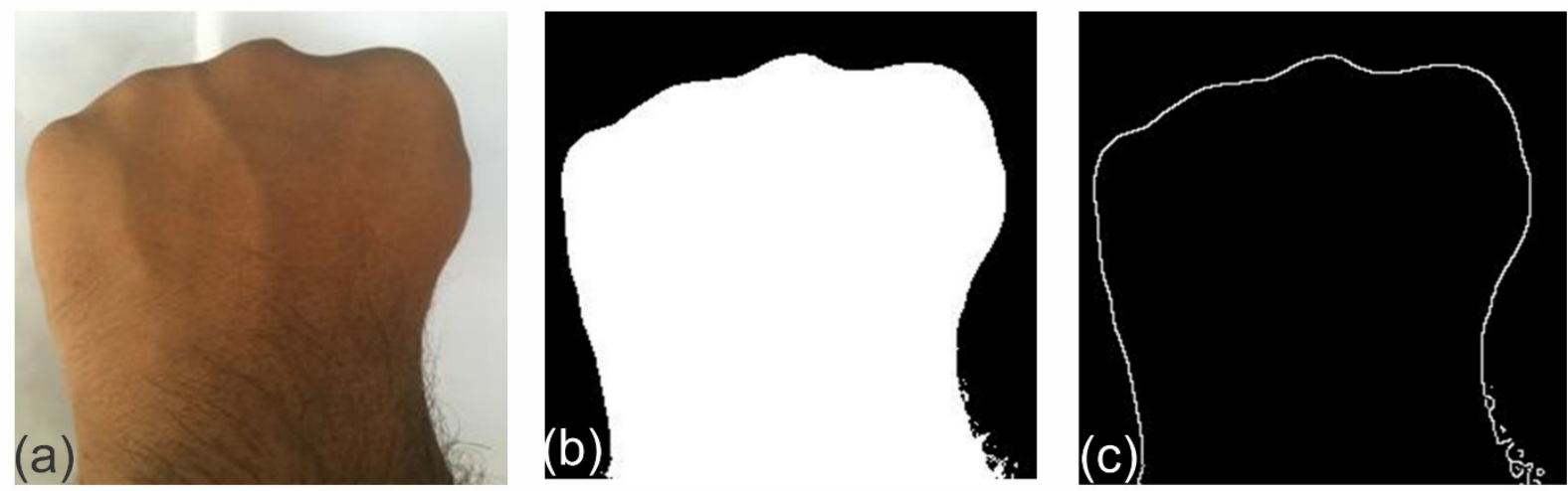

Fig. 3 (a) Input image (b) Binarized image and (c) Edge detected output image

\subsection{Feature extraction}

The knuckle points [7] can be identified by scanning the contour image from left to right row-wise. The order for knuckle identification is in such a manner that first, the middle finger knuckle, second, the index finger knuckle and third, the ring finger knuckle. During scanning, the maximum value of white pixels for each finger is found out, and the corresponding $\mathrm{x}$ and y co-ordinate is extracted. Fig. 4 shows the knuckle point identified for the middle finger, index finger knuckle and the ring finger.

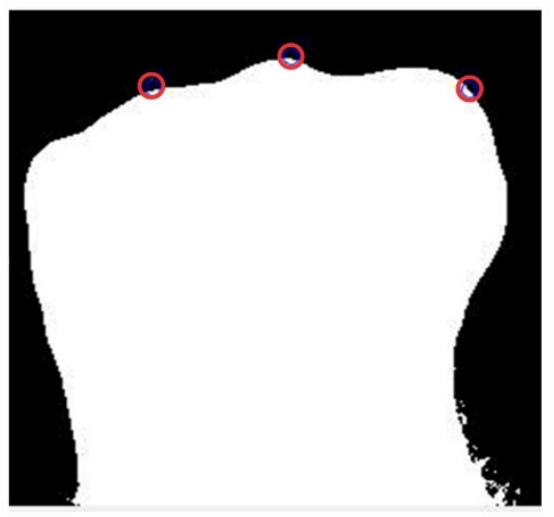

Fig. 4 Knuckle points for the middle finger, index finger knuckle and for the ring finger

After the knuckle point detection, Vein pattern is extracted from input image using maximum curvature method [8]. This method will try to extract the centre lines of the veins. This is done by calculating local maximum curvatures in crosssectional profiles of a vein image. The method is supposed to be robust against varying vein widths and non-uniform brightness. Fig. 5(a) shows the pattern obtained using maximum curvature method. Here the pattern obtained in the figure does not contain all the veins. This is because the imaging device used was not a near-infrared imaging device as visualization of veins is not possible using visible light. The vein pattern extracted is thinned to give one pixel wide output image using MATLAB function 'bwmorph' (Fig 5(b)). The next step is minutia extraction which employs the concept of crossing number [9]. Since veins are continuous there is no ending or termination for veins. The only minutia for veins is vein bifurcations. Fig 5 (c) shows the output image with extracted minutia (bifurcation).

\subsection{UID generation}

Each of the bifurcation extracted is then represented by their position coordinate. "regionprops " and "Centroid” function in Matlab [10] is used to find out the $(\mathrm{x}, \mathrm{y})$ position coordinates of the of the bifurcation. Further, from each of the knuckle points identified, distance to the bifurcation points is calculated using Euclidean distance metric [11]. Using the numerical value thus obtained from hand vein pattern a $4 \mathrm{X} \mathrm{N}$ unique matrix is generated. This $4 \mathrm{X} \mathrm{N}$ matrix of the vain patterns is 
then encrypted to ciphertext using RSA cryptosystem. [12]. During encryption, the private key of the document issuing authority is taken to generate a ciphertext.The ciphertext is provided as input to a QR code generating software such as QR Code studio 1.0 by TEC-IT [12] to generate the corresponding barcode. This QR code serves as the unique ID that can be integrated to the valuable documents to prevent the identity theft. Fig.6 shows the schematic representation of encryption and conversion of the matrix containing the numerical values of the chaotic element to QR code.

(a)

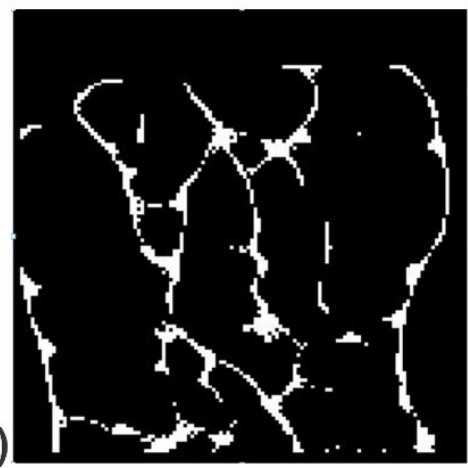

(b)

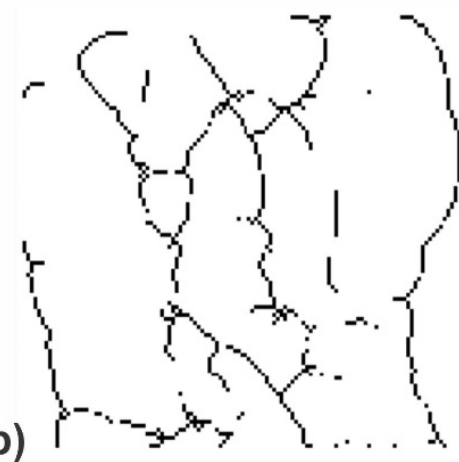

(c)

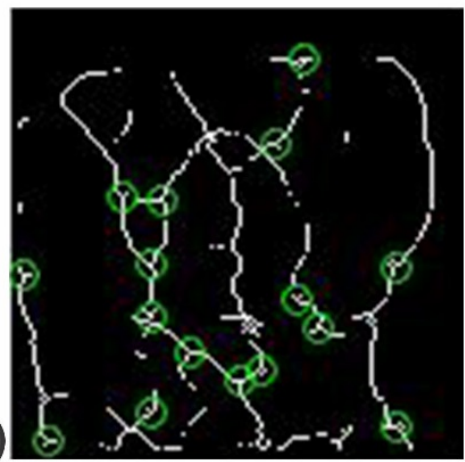

Fig. 5(a) shows the pattern obtained using maximum curvature method (b) the vein pattern after thinning operation (c) the output image with extracted minutia (bifurcation)

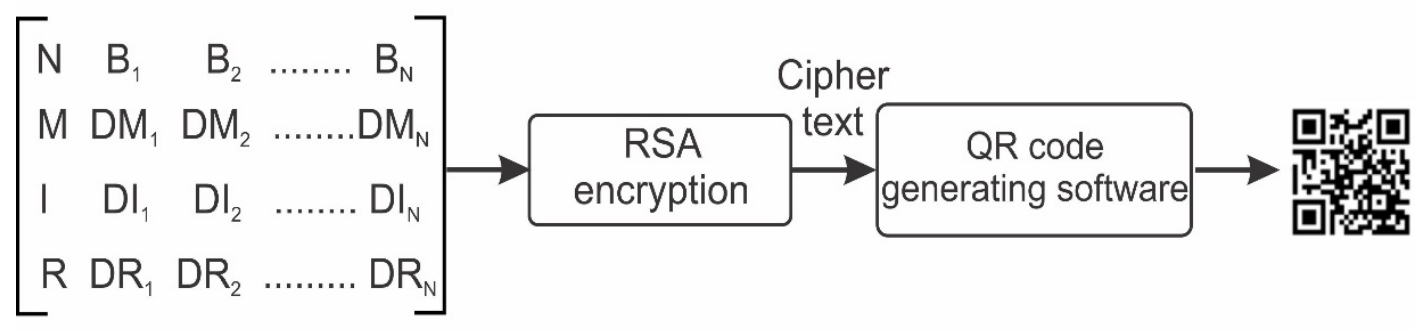

Fig.6 Block diagram of the 4 x N matrix encryption and QR code generation.

Where $\mathrm{M}$, I, and $\mathrm{R}$ represent the (x,y) coordinates of the middle finger, index finger and the ring finger. $\mathrm{N}$ is the total number of bifurcations and B1, B2, and BN represents the (x,y) coordinates of the bifurcations. DM1, DM2. DMN represents the distance values calculated from the knuckle point of the middle finger to the corresponding bifurcations, DI1, DI2........ DIN represents the distance values calculated from the knuckle point of the index finger to the corresponding bifurcations and DR1, DR2 ....... DRN, represents the distance values calculated from the knuckle point of the ring finger to the corresponding bifurcations.

\subsection{Unique ID Verification}

During verification, the QR code embedded valuable document is scanned and stored in a computer. The QR code is read to obtain the ciphertext from the scanned document using a QR code reading software. This ciphertext is then decrypted using the public key, and the matrix or the array of the corresponding numerical value is recovered. At the same instant, the dorsal vein pattern of the person holding the document is captured, and the numerical value is also extracted using the same procedure as explained above. Finally, a comparison is made between the numerical value obtained from the captured image and decrypted the QR code. If they are matching, then the document is authentic.

\section{ANALYSIS AND DISCUSSION}

Analysis of the developed system is carried out by taking images of the dorsal side of an individual's hand using a regular type mobile camera. To ensure the repeatability of pattern structure and the value of the distance calculated, the distance calculation is done in the dorsal vein pattern at various instants of time. The dorsal vein pattern of the right-hand images of 2 people at four different time instants at a gap of 30 minutes is captured and the distance values from each knuckle 
points to the bifurcations is calculated. Fig. 7 (a) \& (b) shows the distance values calculated from the right-hand vein pattern of the person 1 and person 2 at four-time instants.
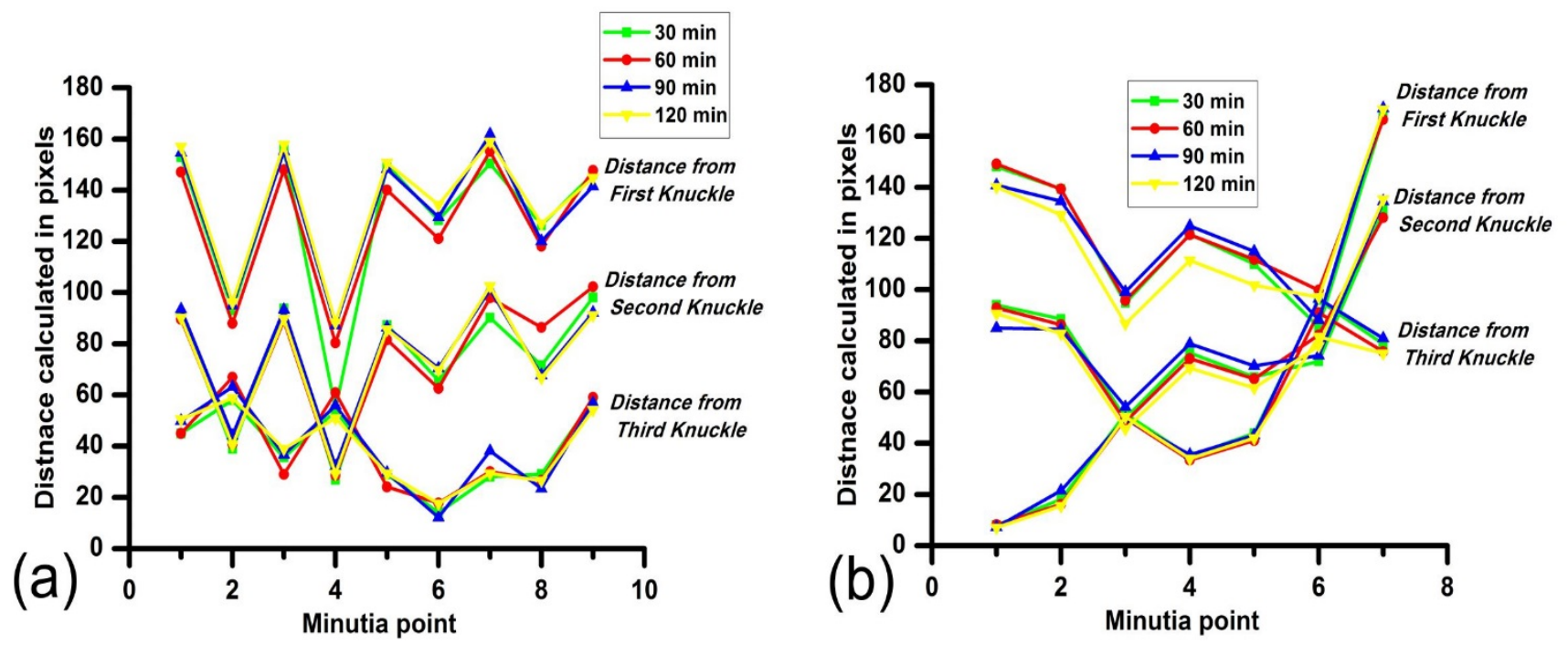

Fig. 7 Distance values calculated for (a) person 1 (b) person 2 at various time instants

From the above analysis, it can be seen that the proposed idea will bring out better results or can be used for identification of person. At the verification stage, a tolerance of \pm 10 is set for the equality checking. Also if the hand veins are captured using the device capable of visualizing veins like an infrared imaging device or a low-cost vein viewer like Cvein [14][15], the tolerance level can be reduced.

\section{CONCLUSION}

This paper proposes an analysis to generate a unique ID for the valuable documents based on the hand vein pattern obtained from the dorsal side of the hand. The proposed method concentrates on the back side of hand as the veins seen here are superficial veins which lie just beneath the skin. Here clenched fist position of the hand is used to extract both knuckle points and minutia features like bifurcation point from the hand to generate a QR code. The unique ID thus formed can be printed on the valuable documents to prevent criminal impersonation. Analysis of the proposed system shows that better results can be obtained by using IR imaging devices like vein viewer, and Cvein. Nowadays used by doctors in hospitals for visualizing veins of patients for treatments of vein disorders. These devices available in markets now can view veins of only a small portion. The future work includes developing vein viewer capable of capturing the whole dorsal side of the hand along with a webcam fitted, to interface the image captured with the PC. This study established the feasibility for using dorsal hand vein pattern as another biological item for generating unique ID (UID) for preventing identity theft in the valuable documents.

\section{REFERENCES}

[1] S. Ambadiyil, Haritha Sree G S and V. P. M. Pillai, "Facial periocular region based unique ID generation and one to one verification for security documents," 2016 2nd International Conference on Advances in Electrical, Electronics, Information, Communication and Bio-Informatics (AEEICB), Chennai, India, 2016, pp. 558-562.doi: 10.1109/AEEICB.2016.7538353

[2] C. Nandini, Ashwini C, Medha Aparna, Niveditha Ramani and Sheeba .K, "Biometric Authentication by Dorsal Hand Vein Pattern", International Journal of Engineering and Technology, volume 2, No.5, May 2013. 
[3] Annemarie Nadort," The Hand Vein Pattern Used as a Biometric Feature", Master Literature Thesis, Amsterdam May 2007.

[4] Chih-Bin Hsu, Shu-Sheng Hao, Jen-Chun Lee, "Personal authentication through dorsal hand vein patterns," Optical Engineering 50(8), 087201 (1 August 2011). https://doi.org/10.1117/1.3607413.

[5] Ajay Kumar, K. Venkata Prathyusha, Personal Authentication using Hand Vein Triangulation and Knuckle Shape, IEEE Transactions on Image Processing vol. 38, pp. 2127-2136, September 2009.

[6] Lin, C. L. and Fan, K. C. Biometric Verification Using Thermal Images of Palm-Dorsa Vein Patterns. IEEE Transactions on ciruits and systems for video technology 14, 199 - 213, 2004.

[7] Amit P. Sahu, Bharathi H.N, "Veins based Authentication System", International Journal of Computer Applications (0975 - 8887) Volume 120 - No.20, June 2015.

[8] Wan Nourul Akmal Ab Aziz, Kamaruzzaman Seman, Ismail Abdullah, M. Norazizi Sham Mohd Sayuti, "Finger Vein Minutiae Points Extraction based on Maximum Curvature Points in Image Profile and Finger Print Application Methods", Australian Journal of Basic and Applied Sciences, 7(8): 751-756, 2013, ISSN 1991-8178.

[9] Ravi. J, K. B. Raja, Venugopal. K. R, “Fingerprint Recognition Using Minutia Score Matching”, International Journal of Engineering Science and Technology, 1(2) ( 2009).

[10] http://www.mathworks.in/help/images/ref/regionprops.html\#bqkf8ln

[11] Sunita Aeri and Sukhvinder Kaur, " Vein Patterns as Bio-Metric Identifier using Euclidean Distance", International Journal of Computer Applications (0975 - 8887) Volume 89 - No 20, March 2014

[12]William Stallings, “Cryptography and Network Security Principles and Practices”, Prentice Hall, 2005.

[13] http://www.tec-it.com/en/start/Default.aspx

[14] Aiken, Rachel Sarah, " Vein Pattern Analysis - A comparison of two vein imaging modalities", Master Of Science, Award date: 2014.

[15] Kousain Raza Beg, Mantesh Vhadalure, Sumeet Jaokar, Abhishek Saiawani, "Vein Structure Authentication System", MPGI National Multi Conference, 2012. 